Advanced AAM 기반 정서특징 검출 기법 개발

\title{
Development of Emotional Feature Extraction Method based on Advanced AAM
}

\author{
고광은 · 심귀보* \\ Kwang-Eun Ko and Kwee-Bo Sim ${ }^{*}$ \\ 중앙대학교 전자전기공학부
}

요 약

지능로봇시스템과 같은 $\mathrm{HCI}$ 환경에서 사람의 감정을 인식하기 위한 매개정보인 얼굴영상 기반 정서특징 검출문제는 관련 분야의 매우 중요한 이슈이다. 본 논문에서는 보편화된 시스템 기반에서 임의의 사용자에 대한 정서 인식을 수행하기 위해 사람의 얼굴에서 나타나는 최적의 정서특징을 가장 효율적으로 추출하기 위한 연구로서 본 연구실에서 기존에 제안한 $\mathrm{FACS}$ 와 AAM을 이용한 Bayesian Network 기반 얼굴표정 인식 시스템을 보완한 Advanced AAM을 기반 얼굴영상 정서 특징을 검출 시스템에 대한 연구를 진행하였다. 이를 수행하기 위하여 정규화된 이미지에서의 Statistical Shape Analysis 로서 Advanced AAM과 얼굴 표정 분석 시스템인 $\mathrm{FACS}$ 를 이용하여, 임의의 사용자에 대한 자동적인 정서특징 검출이 가 능하도록 연구를 진행하였다.

\begin{abstract}
It is a key element that the problem of emotional feature extraction based on facial image to recognize a human emotion status. In this paper, we propose an Advanced AAM that is improved version of proposed Facial Expression Recognition Systems based on Bayesian Network by using FACS and AAM. This is a study about the most efficient method of optimal facial feature area for human emotion recognition about random user based on generalized HCI system environments. In order to perform such processes, we use a Statistical Shape Analysis at the normalized input image by using Advanced AAM and FACS as a facial expression and emotion status analysis program. And we study about the automatical emotional feature extraction about random user.
\end{abstract}

Key Words : AAM, ASM, LDA, FACS, Feature Extraction

\section{1. 서 론}

자동적인 얼굴인식, 표정 분석, 이미지 복구, 감정인식 등 Human-Computer Interface 시스템과 관련된 분야에 대한 연구가 활성화되고 있으며, 많은 연구결과들이 제시되 고 있다. 이 중에서도 사람의 얼굴 영상 분석을 활용한 $\mathrm{HCI}$ 시스템은 구현의 용이성뿐만 아니라 얼굴 영상이 내포하고 있는 다양한 정보(감정, 심리반응, 생체반응 등)로 인하여 그 효용성이 초기부터 주목받고 있으며, 공학뿐만 아니라 심리학과 같은 인문 분야에서도 널리 쓰인다.

심리학에서 독립적인 얼굴 표정을 기반으로 6 개의 기본 정서를 제시한 Ekman의 경우, 제시된 6가지 정서를 기본 6 정서(기쁨, 슬픔, 분노, 공포, 혐오, 놀람)로 규정하여 보편적 인 얼굴영상 분석 시 활용할 수 있도록 하는 연구결과를 발 표하였다[1].

그러나 공학에서는 기존의 단순 얼굴표정 분석에서 한

접수일자 : 2009년 11월 09일

완료일자 : 2009년 11월 26일

* 교신 저자

이 논문은 2009년도 정부(교육과학기술부)의 재원으로 한국연구재단의 지원을 받아 수행된 연구입니다(No. 2008-0060738). 연구비지원에 감사드립니다.
단계 더 나아가, 입력된 영상을 활용하여 자동적으로 얼굴 을 추적하거나 인식할 수 있는 시스템화 된 결과가 요구된 다. 이러한 연구는 보안카메라, 로봇 등에 적용됨으로써 침 입자 추적, 보안 얼굴인식, 영상분석 기반 엔터테인먼트에 활용하는 등, 다양한 응용 시스템의 개발로 이어질 수 있다.

이와 같이, 사람의 얼굴영상을 활용한 다양한 분석 시 선 행되어져야하는 과정이 바로 얼굴 특징 검출에 있는데, 본 논문에서 주로 다루고자 하는 특징은 사람의 감정을 표현함 에 있어 밀접한 연관을 가지는 정서적 특징 검출에 초점을 맞추어 논하고자 한다. 얼굴영상에서 특징을 추출함에 있어 서 그 입력의 다양성으로 인하여 여러 가지 방법이 개발되 어져 왔다.

우선, Statistical Model에 기반으로 얼굴영상에 존재하 는 다양한 특징들을 추적하는 알고리즘인 $\mathrm{ASM}$ (Active Shape Model), AAM(Active Appearance Model)은 Cootes에 의하여 제안되었으며 Training Set을 기반으로 Deformable Model을 생성함으로써 얼굴인식 및 추적에 활 용 가능한 특징을 검출하는 방향으로 많이 쓰인다[2].

또한 M. Kass에 의해 1987년 발표된 Snakes: $\mathrm{ACM}$ (Active Contour Model) 알고리즘은 입력된 영상에 서 주요한 특징이 되는 가장자리(Edge) 검출을 위하여 곡 면의 에너지를 최소화 시키는 Spline을 탐색하는 알고리즘 이다[3.] 
이밖에도 열화상카메라로 촬영한 적외선 얼굴영상에서 특징영역 검출을 위한 알고리즘으로써 3D 얼굴 Deformable Model을 생성하여 적외선 이미지에 적용하여 특징검출기법 이 제시된 바 있다[4].

본 논문에서는 상기에 언급된 알고리즘 중 $\mathrm{AAM}$ 을 활용 한 특징 검출을 설명하고, 기존의 방법에서 초기 설정에 대 하여 강인한(Robust) 특성을 가지는 방향으로 보완한 Advanced-AAM을 제시한다. 그리고 FACS(Facial Action Coding System)과의 융합을 통해 효율적으로 얼굴 영역에 서의 정서특징을 검출 할 수 있도록 하는 방법을 모색한다.

\section{2. 관련 선행 연구}

\section{1 영상 기반 특징 검출 선행 연구 내용}

앞서 언급된 여러 가지 특징 검출 알고리즘 중 본 논문에 서는 $\mathrm{AAM}$ 을 기본으로 활용한 특징 검출기법을 제안한다.

본 연구 이전에 주로 사용된 특징 검출 방식은 Static/Dynamic 특성을 가지는 특징에 대한 고려에서 시작 하여 Dynamic의 경우 Optic flow를 이용하여 얼굴특징을 추출하는 방식과[5], Static의 경우, 얼굴영역과 비 얼굴영역 분포의 패턴을 인식하여 그에 따른 국소 이미지 패턴 분포 기반 모델을 통해 특징 벡터를 추출하는 방식을 주로 활용하 였다. 이와 관련된 대표적인 사례가 $\mathrm{PCA}$ (Principal Component Analysis)를 이용한 고유 얼굴(Eigenface) 알고 리즘이다[6]. Eigenface는 얼굴인식 및 분류에 주로 쓰이며 컴퓨터 비전 프로그램 내의 Training Set의 Eigenvector의 집합으로 표현된다. Training Set을 구성하고 있는 $M$ 개의 영상의 크기가 $N \times N$ 일 때, 각 영상을 $M$ 개의 $N^{2} \times 1$ 행벡 터로 변환한 집합 $S$ 는 다음과 같다.

$$
S=\left\{\Gamma_{1}, \Gamma_{2}, \Gamma_{3}, \ldots \ldots, \Gamma_{M}\right\}
$$

입력 영상에 대한 Normalization 과정을 가정하고 평균 영상과 각 Training Set의 이미지와의 편차를 계산하면 다 음과 같다.

$$
\begin{gathered}
\Psi=\frac{1}{M} \sum_{m=1}^{M} \Gamma_{m} \\
\Phi_{i}=\Gamma_{i}-\Psi
\end{gathered}
$$

구해진 편차로부터 Training Set에 대한 Covariance Matrix를 계산하면 다음과 같다.

$$
\begin{gathered}
C=\frac{1}{M} \sum_{n=1}^{M} \Phi_{n} \Phi_{n}^{T}=A A^{T} \\
A=\frac{1}{\sqrt{M}}\left[\Phi_{1}, \Phi_{2}, \Phi_{3}, \ldots, \Phi_{M}\right]
\end{gathered}
$$

이렇게 구해진 Covariance Matrix, $C$ 에 대한 Eigenvalue와 Eigenvector를 구하여 Eigenvalue에 대한 Eigenrvector를 $N \times N$ 으로 재배열하면 입력영상과 유사한 이미지가 나오는데, 이를 Eigenface라 하고 이를 수식으로 표현하면 다음과 같다.

$$
u_{l}=\frac{1}{M} \sum_{k=1}^{M} \lambda_{l k} \Phi_{k}, \quad l=1,2, \ldots, M
$$

Eigenface 기반의 특징 검출 방식은 입력 이미지에 대한
전체적인 특징을 검출하여 활용함으로써 얼굴인식, 추적 등 의 활용에 적합한 특성을 가진다. 그러나 얼굴 이외의 영역, 즉 외란(Noise)에 대하여 민감하고, 정서인식과 같이 국소 적인 특징(표정)영역에서의 정보해석이 요구되는 경우 Eigenface를 통해 검출된 특징 정보는 결과의 효율성과 정 확성이 떨어진다고 볼 수 있다.

따라서 본 논문에서는 $\mathrm{AAM}$ 을 기본으로 하는 특징 검출 기법을 활용하여 연구를 진행하였다.

\subsection{Traditional Active Appearance Model}

Active Appearance Model(이하, AAM)은 영상 또는 이 미지에 대한 특징 검출을 위한 통계적인 모델링 기법으로 높은 유동성(Flexible)을 가지는 Deformable Model을 활용 하여 이미지 모델링을 가능하게 한다[2]. AAM은 Cootes에 의하여 1998년 제안되었으며[7] 이전 버전인 ASM(Active Shape Model) 기본으로 하여 ASM의 외란, 얼굴 움직임 등에 민감하다는 단점을 보완하였다[8].

$\mathrm{AAM}$ 을 구성하는 요소는 크게 두 가지로 Shaep Model 과 Texture Model이다. Shape, Texture Model 정보를 이 용하여 객체의 Shape 형태를 유지함과 동시에 학습된 Texture 정보를 기반으로 가장 유사한 Texture를 가진 영 역을 검색하여 보다 정확하게 객체의 모양과 위치를 탐색 가능하게 한다.

Shape 모델은 목표(Target)가 되는 객체(Object)에 대하 여 Translation, Rotation, Scaling 효과가 제거된 형태의 모든 기하학적 정보를 일컬으며 Training Set의 이미지 전 체에 대하여 Shape Model 하기 위한 과정이 필요하다.

Training Set에 속하는 이미지들의 $n$ 개의 Landmark를 찍어 특징점을 가지는 Shape를 가정한다면, 각각의 이미지 는 Shape $X$ 로서 다음과 같은 벡터 형식으로 표현 가능하다.

$$
X_{i}=\left[x_{i 1}, x_{i 2}, \ldots . ., x_{n}, y_{i 1}, y_{i 2}, \ldots \ldots, y_{n}\right]^{T}
$$

또한, Training Set 전체에 대한 평균과 각각의 Shape에 대한 편차를 구하면 다음의 식과 같다.

$$
\begin{gathered}
\bar{X}=\frac{1}{N} \sum_{i=1}^{N} X_{i} \\
d X_{i}=X_{i}-\bar{X}
\end{gathered}
$$

앞서 언급된 Eigenface를 구할 때 사용된 $\mathrm{PCA}$ 를 사용하 여 $d X_{i}$ 의 Covariance Matrix $C_{s}$ 와 $C_{s}$ 의 Eigenvalue를 구 하고 그에 대응되는 Eigenvector를 구한 결과는 다음과 같다.

$$
\begin{aligned}
C_{s} & =\frac{1}{N} \sum_{i=1}^{N} d X_{i} d X_{i}^{T}, \quad N \text { 은 shape 개수 } \\
\lambda_{s} & =\left[\begin{array}{ccc}
\lambda_{1} & 0 & 0 \\
0 & \ddots & 0 \\
0 & 0 & \lambda_{2 n}
\end{array}\right], \Phi_{s}=\left[\phi_{1}, \phi_{2}, \ldots, \phi_{2 n}\right]
\end{aligned}
$$

이 때, Shape의 평균과 Eigenvector $\Phi_{s}$ 의 선형 조합을 통해 기존의 Training Set을 기반으로 표현 가능한 선형 모 델을 다음과 같이 나타낼 수 있다.

$$
\begin{gathered}
X=\bar{X}+\Phi_{s} b_{s} \\
b_{s}=\Phi_{s}^{T}(X-\bar{X})
\end{gathered}
$$


$b_{s}$ 는 Shape Model 표현을 위하여 사용되는 Pose 파라미 터이다.

아래의 그림 1은 이러한 Shape Model 과정을 통해 생성 된 결과를 도시하고 있다.

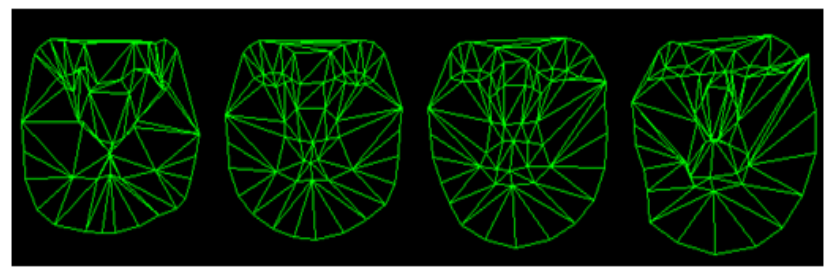

그림 1. Shape Model 생성

Fig. 1. Creation of Shape Model

Texture 모델은 추출하고자 하는 특징 영역에 대한 이미 지에서의 Pixel Intensity를 말하며, Shape 모델이 객체의 특징점들 간의 위치관계만을 고려하는 것에 반하여 Pixel Intensity를 고려함으로써 특징 추출 성능 향상을 기대할 수 있다. Texture 모델은 Shape와 달리 조명, 광원(光源) 효과에 민감하므로 이를 최소화하기 위한 Normalization 과정을 거쳐야 한다. Scaling factor : $\alpha$, offset factor : $\beta$ 라 할 때, Norm. 과정은 아래 수식과 같다.

$$
g=\frac{g_{i m}-\beta^{*} 1}{\alpha}, g_{i m}: \text { 이미지 Texture data }
$$

Norm. 과정을 거친 Texture data $g$ 에 대하여 Shape 모 델과 동일한 $\mathrm{PCA}$ 과정을 통해 선형적인 모델을 구한다면 다음의 식과 같다.

$$
g=\bar{g}+\Phi_{g} b_{b}
$$

$\Phi_{g}$ 는 Shape 모델의 경우와 동일하게 Texture의 분포에 대하여 $\mathrm{PCA}$ 를 적용한 Eigenvector의 집합이며 $b_{g}$ 는 Texture 모델을 표현하기 위한 파라미터이다. $b_{g}$ 의 표현은 다음과 같이 표현할 수 있다.

$$
b_{g}=\Phi_{g}^{T}(g-\bar{g})
$$

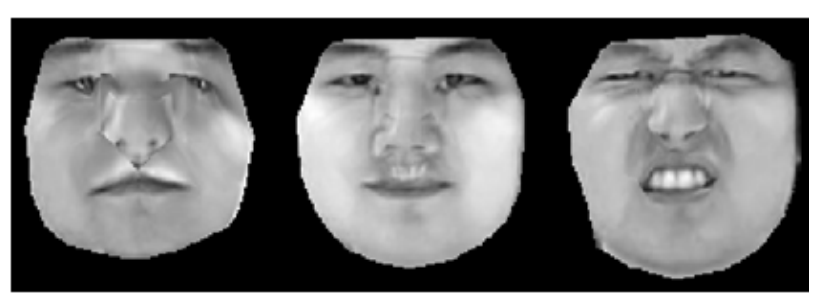

그림 2. Texture Model 생성

Fig. 2. Creation of Texture Model

\subsection{Facial Action Coding System}

FACS는 Paul Ekman과 Wallace Friesen에 의해 1976 년 제안된 얼굴 표정 분석 기법으로 해부학적 토대로 사람 의 얼굴 근육에 대한 해부학적인 분석을 통하여 46개의 Action Unit을 설정하고 이를 기반으로 얼굴표정을 분석할 수 있다[9]. 아래의 표 1은 Action Unit에 대한 정의와 관련 된 얼굴 근육 영역을 나타내고 있다[10].

표 1 의 Action Unit은 감정 표현 시 움직이는 얼굴 근육
에 대한 묘사로 기존의 $\mathrm{FACS}$ 를 활용한 연구는 다수의 표 정을 나타내는 얼굴이미지에 대하여 수동으로 Action Unit 을 비교, 분석하여 어떤 표정을 표현하고 있는 결정하는 일 종의 Manual System이다. 그러나 FACS를 적용하여 자동 으로 얼굴 기반 정서특징 검출을 위하여 본 논문에서는 패 턴분류 알고리즘과의 융합을 통해 사람의 표정에서 나타나 는 정서 특징을 쉽게 추출할 수 있는 정서특징 검출 시스템 을 개발하고자 하는 것이다.

\begin{tabular}{|c|c|c|c|c|c|}
\hline $\mathrm{AU}$ & Description & Facial muscle & $\overline{A U}$ & Description & Facial muscle \\
\hline & Inner Brow Raiser & Frontalis, pars medialis & 22 & Lip Funnelet & Orbicularis oris \\
\hline 2 & Outer Brow Raiser & Frontalis, pars lateralis & 23 & Lip Tightener & Orbicularis oris \\
\hline 4 & Brow Lowerer & $\begin{array}{l}\text { Corrugator supereilii, } \\
\text { Depressor superciliii }\end{array}$ & $\underline{24}$ & Lip Pressor & Orbicularis on is \\
\hline$\underline{5}$ & Upper Lad Raiser & Levator palpebrae superioris & $\underline{25}$ & Laps part"* & $\begin{array}{l}\text { Depressor labii inferioris of relaxation } \\
\text { of Mentalis, or Orbicularis oris }\end{array}$ \\
\hline$\underline{6}$ & Check Raiget & Otbicularis oculi, pars orbitalis & $\underline{26}$ & Jaw Drop & $\begin{array}{l}\text { Masseter, relaxed Temporalis and } \\
\text { intemal Prerygoid }\end{array}$ \\
\hline 7 & Lid Tightener & Orbieularis bectif, paspalpebralis & 27 & Mouth Stretch & Pterygoids, Digastric \\
\hline$\underline{2}$ & Nose Wrinkler & $\begin{array}{l}\text { Levator labii superioris } \\
\text { alaquae nass }\end{array}$ & 28 & Lip Suck & Orbicularis oris \\
\hline$\underline{10}$ & Upper Lip Raiser & Levator labii superioris & 41 & Lid droop & $\begin{array}{l}\text { Relaxation of Levator palpebrae } \\
\text { superioris }\end{array}$ \\
\hline 11 & $\begin{array}{l}\text { Nasolabial } \\
\text { Deepener }\end{array}$ & Zygomaticus minor & 42 & Slit & Orbicularis oculi \\
\hline 12 & $\begin{array}{l}\text { Lip Coneter } \\
\text { Pullet }\end{array}$ & Zygomaticus major & 43 & Eyes Closed & \begin{tabular}{|l} 
Relaxation of Levatior palipebrat superi- \\
oris; Orbscularis oculi, pars palpectratis
\end{tabular} \\
\hline 13 & Cheek Puffer & $\begin{array}{l}\text { Levator anguli oris } \\
\text { (a.k. Caninus) }\end{array}$ & 44 & Squint & Orbicularis oculi, pars palpebralis \\
\hline 14 & Dimplet & Buccinator & 45 & Blink & $\begin{array}{l}\text { Relaxation of Levator palpebra super } \\
\text { oris: Otbicularis oculit pars palpebratis }\end{array}$ \\
\hline 15 & $\begin{array}{l}\text { Lip Corner } \\
\text { Depressor }\end{array}$ & $\begin{array}{l}\text { Depressor angul orts } \\
\text { (a.ka. Triangularis) }\end{array}$ & 46 & Wimk & 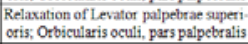 \\
\hline 16 & $\begin{array}{l}\text { Lower Lip } \\
\text { Depressor }\end{array}$ & Depressor labu inferioris & & & \\
\hline 17 & Chin Raiser & Mentalis & & & \\
\hline 18 & Lip Puckerer & $\begin{array}{l}\text { Incisiviui labii superioris and } \\
\text { Incisivin labbrn infertioris }\end{array}$ & & & \\
\hline 20 & Lip stretcher & Risorius w/ platysma & & & \\
\hline
\end{tabular}

표 1. Facial Action Coding Systems

Table 1. Facial Action Coding Systems

\section{3. 제안 알고리즘 및 기술}

\subsection{Linear Discriminant Analysis}

Linear Discriminant Analysis(이하, LDA)는 PCA와 함 께 대표적인 특징 벡터 차원 축소 기법 중 하나로, 특징 공 간상에 존재하는 다수의 클래스를 가정하여 클래스 간 분산 과 클래스 내 분산의 비율을 최대화하는 방식으로 데이터에 대한 특징 벡터의 차원을 축소시킨다[12]. LDA는 특징 벡 터의 차원 축소를 통해 분산된 클래스를 분류하는 분류기 (Classifier)의 역할을 수행할 수 있다[13].

Sample 데이터 벡터를 $X$, 투영(Projection)시키고자 하 는 주성분 축(Principal Axix)를 $W$ 라 하고 수식을 전개하 면 다음과 같다.

$$
Y=W^{T} X
$$

그리고 $\omega_{i}$ 에 속하는 $N$ 개의 샘플들의 원 공간에서 평균 과 투영 후 평균을 정의하면 다음과 같다.

$$
\begin{gathered}
m_{i}=\frac{1}{N} \sum_{X \in \omega_{i}} X \\
\overline{m_{i}}=\frac{1}{N} \sum_{Y \in \omega_{i}} Y=\frac{1}{N} \sum_{Y \in \omega_{i}} W^{T} X=W^{T} m_{i}
\end{gathered}
$$

위 식 (17)과 (18)을 통해 투영 데이터 중심 간의 거리를 공식화 하면 다음과 같다.

$$
\begin{aligned}
\left|\overline{m_{1}}-\overline{m_{2}}\right| & =\left|W^{T} m_{1}-W^{T} m_{2}\right| \\
& =\left|W^{T}\left(m_{1}-m_{2}\right)\right|
\end{aligned}
$$


식 (19)의 결과를 목적함수로 하는 경우 투영된 벡터의 중심 간 거리가 클래스 간의 분산을 고려하지 않기 때문에 이를 고려한 $\mathrm{LDA}$ 의 핵심은 결국 평균 간의 차이를 Normalization하여 함수를 표현하고 이 목적함수를 최대화 하는 방식을 제안하였다. 각각의 클래스에 대한 분산을 정 의하면, 다음과 같다.

$$
\widetilde{s_{i}^{2}}=\sum_{Y \in \omega_{i}}\left(Y-\widetilde{m_{i}}\right)^{2}
$$

그리고 $\left(\widetilde{s_{1}}+\widetilde{s_{2}}\right)$ 을 사영표본의 클래스 내 분산이라고 한 다. $\mathrm{LDA}$ 의 목적은 분류를 위해 필요한 목적함수를 최대화 하기 위한 $W$ 를 찾는 것이다. 목적함수 $J(W)$ 는 다음과 같다.

$$
J(W)=\frac{\left|\widetilde{m_{1}}-\widetilde{m_{2}}\right|^{2}}{\left(\widetilde{s_{1}^{2}}+\widetilde{s_{2}^{2}}\right)}
$$

식 (20)을 분산 행렬이 포함된 함수로 다시 표현 하면 다 음과 같다.

$$
\begin{gathered}
\widetilde{s_{i}^{2}}=\sum_{X \in \omega_{i}}\left(W^{T} X-W^{T} m_{i}\right)^{2}=W^{T} S_{i} W \\
\text { 단, } S_{i}=\left(X-m_{i}\right)\left(X-m_{i}\right)^{T}
\end{gathered}
$$

이 때, $\tilde{s}_{1}^{2}+\tilde{s}_{2}^{2}=W^{T} S_{W} W$ 가 성립하고, 마찬가지로 $\left(\widetilde{m_{1}}-\widetilde{m}_{2}\right)^{2}=W^{T} S_{B} W$ 일 때, $S_{W}, S_{B}$ 를 클래스 내 분산, 클 래스 간 분산으로 정의할 수 있다.

최종적으로 앞서 $J(W)$ 를 $S_{W}, S_{B}$ 의 항으로 재정의한다 면 다음과 같다.

$$
J(W)=\frac{W^{T} S_{W} W}{W^{T} S_{B} W}
$$

이 식 (23)의 목적함수를 최대화 하는 행렬 $W$ 를 구하면 우리가 구하고자 하는 선형 분류가 가능해진다. 이를 수행 하기 위하여 일반화된 고유 값 문제의 해법을 이용하는 수 식을 적용하면 다음과 같은 결과가 나온다.

$$
W=\arg \max _{W} \frac{W^{T} S_{B} W}{W^{T} S_{W} W}=S_{W}^{-1}\left(m_{1}-m_{2}\right)
$$

위 식을 통해 구해진 행렬 $W$ 를 분류하고자하는 특징 공 간상에 적용하여 변환하면 투영시키고자 하는 축을 기준으 로 선형 분류가 시행된다.

\subsection{Advanced AAM}

기존의 $\mathrm{AAM}$ 의 구축을 통해 검출된 Shape Model과 Texture Model을 기반으로 정서특징 영역을 검출하기 위 한 방법으로 본 논문에서는 $\mathrm{LDA}$ 를 적용하여 추출하고자 하는 특징영역을 검출한다. 앞서 3.1절에서 설명한 바와 같 이 $\mathrm{LDA}$ 는 2 차원 특징 벡터로 이루어진 클래스 간의 분류 를 위한 분류기의 성격을 가지므로 Shape Model에서의 특 징 점에 해당되는 각 Landmark에서 일차적으로 눈 영역과 입 영역에 해당되는 클래스를 구분 짓는 축을 찾는 과정을 거칠 필요가 있다.

\section{$3.3 \mathrm{FACS}$ 를 이용한 정서 특징 분석}

$\mathrm{AAM}$ 을 통해 정서인식을 위해 필요한 Shape, Texture Model의 생성 이후, 그 결과를 통해 올바른 특징 분석, 즉 정서인식을 수행하기 위하여 필요한 것은 신뢰성 있는 얼굴 표정 분석 기법의 적용이다. 이는 앞서 설명한 2.3절의 $\mathrm{FACS}$ 를 통해 적용할 수 있으며 Ekman의 기본 6정서에 해당되는 감정 표현 시 반응하는 안면 근육에 대응되는 주 요 Action Unit만을 선별하여, 모델링함으로써 올바른 인식 결과가 도출될 수 있도록 한다. 다음 표 2는 기본 6정서에 기반으로 본 논문의 시뮬레이션 과정을 통해 활용되는 주요 Action Unit에 대한 정의를 나타내고 있다.

표 2. 기본 6정서 기반 주요 Action Unit

Table 2. Main Action Unit based on 6 basic emotion

\begin{tabular}{|c|c|c|c|}
\hline AU \# & Definition & AU \# & Definition \\
\hline 1 & Eyebrow Lower & 5 & Eyelid Lower a little \\
\hline 2 & Eyebrow Raiser & 6 & Mouth Corner Raiser \\
\hline 3 & Eyelid Raiser a little & 7 & $\begin{array}{c}\text { Mouth Corner } \\
\text { Lower }\end{array}$ \\
\hline 4 & Eyelid Raiser a lot & 8 & Mouth Open \\
\hline
\end{tabular}

\section{Advanced-AAM 기반 정서 특징 검출 시뮬레이션}

$\mathrm{LDA}$ 에 의해 선별된 특징 영역 클래스에 대한 $\mathrm{FACS}$ 의 적용을 통해 정서인지결과를 도시하기 위한 시뮬레이션으 로써 Bayesian Network를 통한 확률 기반 접근을 수행하 고자 한다[14].

The Technical University of Denmark에서 제공하는 AAM Application과 AAM-API[15]를 통해 시뮬레이션 프 로그램을 작성하고 그림 3 과 같이 입력영상에 대한 $\mathrm{AAM}$ 을 구축하는 과정을 통해 정서특징을 지니는 얼굴 영역에 대한 검출을 시행하였다.

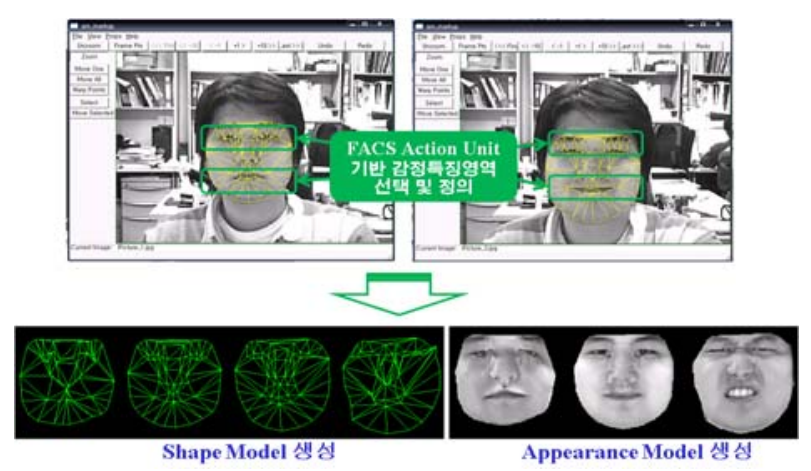

그림 3. Input Image에 대한 AAM 구축

Fig. 3. Structure of AAM for input image

검출된 $\mathrm{AAM}$ 의 얼굴영역에서 $\mathrm{LDA}$ 를 통해 정서인식을 위해 필요한 Shape, Texture Model 에서의 정서 특징 영 
역을 분류하고 이후, 그 결과를 통해 올바른 특징 분석하기 위하여 FACS를 기반으로 구축된 Bayesian Network로 적 용한 결과가 다음의 그림 4 와 같다.

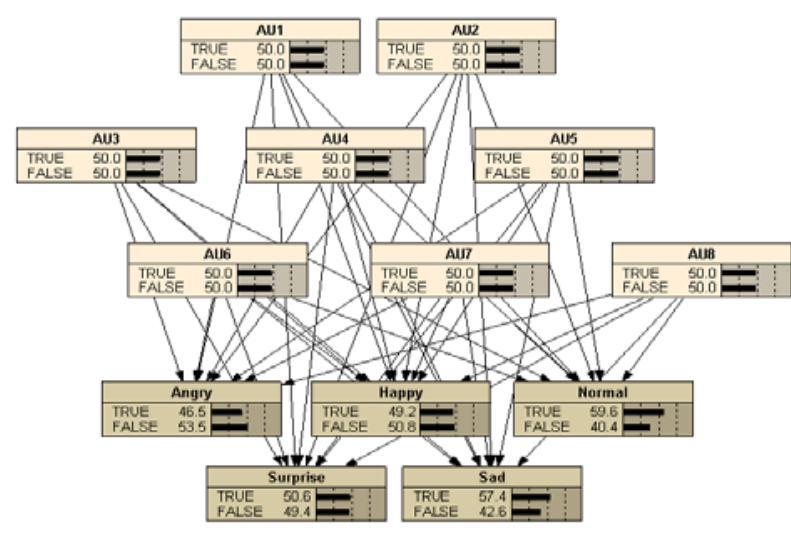

그림 4. FACS 기반 Bayesian Network Structure Fig. 4. FACS based Bayesian Network Structure

그림 4의 $\mathrm{BN}$ 에 입력된 이미지의 $\mathrm{AU} 2, \mathrm{AU} 3, \mathrm{AU} 4, \mathrm{AU} 8$ 에 따른 인지 결과를 다음 그림과 같다.

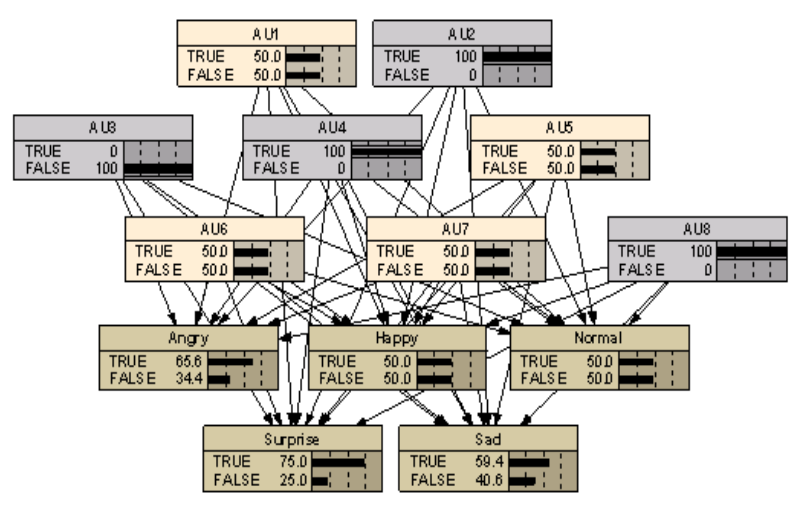

그림 5. Action Unit 변화에 따른 시뮬레이션

Fig. 5. Simulation based on change of Action Unit

\section{5. 결론 및 향후 과제}

본 논문에서는 얼굴 표정에서 나타나는 감정을 인식하기 위한 특징검출 기법으로써 $\mathrm{LDA}$ 와 $\mathrm{AAM}$ 의 융합을 기반으 로 하는 Advanced AAM과 FACS와의 연동을 통해 적절 한 정서특징을 검출하는 기법에 대하여 제안하였다. $\mathrm{LDA}$ 에 의한 선형 분리로 특징 벡터 영역에서의 클래스 간 분류 가 이루어져, 신뢰성 높은 얼굴 감정 특징 영역이 FACS에 적용되었고 이 결과를 Bayesian Network의 결과로 적용하 여, 확률 기반 추론 수행을 통한 정서인지 결과를 분석하게 된다.

향후 추가적인 연구과제로는 정서특징 영역이 비선형적 인 경우를 고려할 수 있도록 $\mathrm{LDA}$ 이외의 새로운 패턴분류 알고리즘을 적용하여 성능을 비교, 평가할 수 있어야 할 것 이며, AAM을 통해 구축하고자 하는 Shape Model과
Texture Model의 생성을 위하여 자동적으로 Landmarker 생성할 수 있는 알고리즘의 개발이 필요하다. 또한, 정서상 태 분석을 위한 베이지안 네트워크의 구조와 CPT에 대한 적절한 구조학습 및 파라미터 학습이 이루어질 수 있도록 추가 연구를 진행할 예정이다.

\section{참 고 문 헌}

[1] P, Ekman, W. V. Friesen, Constants Across cultrues in the Face and Emotion. Journal of Personality and Social Psychology, vol.17, no.2, pp.124 129, 1971.

[2] T. F. Cootes, C. J. Taylor, Statistical Models of Appearance for Computer Vision, 2004.

[3] M. Kass, A. Witkin, D. Terzopoulos, "Snakes: Active Contour Modesl," International Journal of Conputer Vision, vol.1, no.4, pp.321 331, 1987.

[4] Jian-Gang Wang, Eric Sung, "Facial Feature Extraction in an Infrared Image by Proxy Withe a Visible Face Image", IEEE Transactions on Instrumentation and Measurement, vol.56, no.5, pp.2057 2066, 2007.

[5] 이주호, 양현승, "2차원 전역 특징추출에 기반한 얼 굴표정인식 및 모델 변형," 대한전자공학회 워크샵, 제9권, pp.209 214, 1997.

[6] P. N. Belhumeru, J. P. Hespanaha, D. J. Kriegman, "Eigenfaces vs. Fisherfaces: Recognition Using Class Specific Linear Projection," IEEE Transactions on Pattern Analysis and Machine Intelligence, vol.19, no.7, pp.711 720, 1997.

[7] T. F. Cootes, G. J. Edwards. C. J. Taylor, "Active Appearance Models," IEEE Transactions on Pattern Analysis and Machine Intelligence, vol.23, no.6, pp.681 685, 2001.

[8] Seiji Kobayasho and Shuji Hashimoto, Automated feature extraction of face image and its applications, in: International workshop on Robot and Human Communication, pp. 164 169, 2008.

[9] P. Ekman and W. Friesen. Facial Action Coding System: A Technique for the Measurement of Facial Movement. Consulting Psychologists Press, Palo Alto, 1978.

[10] P. Ekman and W. Friesen. http://www.cs.cmu.edu /afs/cs/project/face/www/facs.htm

[11] A. M. Martinez, A. C. Kak, "PCA versus LDA", IEEE Transactions on Pattern Analysis and Machine Intelligence, vol.23, no.2, pp.228 233, 2001.

[12] 한학용, 패턴인식개론: $M A T L A B$ 실습을 통한 입체적 학습, 한빛미디어, 2005.

[13] 오일석, 패턴인식, 교보문고, 2008.

[14] 고광은, 심귀보, "FACS와 AAM을 이용한 Bayesian Network 기반 얼굴표정인식시스템 개발”, 한국지능시스템학회 논문지, vol.19, no.4, pp.562 567, 2009. 
[15] M. B. Stegmann, Informatics and Mathematical Modelling(IMM), The Technical University of Denmark(DTU), http://www2.imm.dtu.dk/ aam

\section{저 자 소 개}

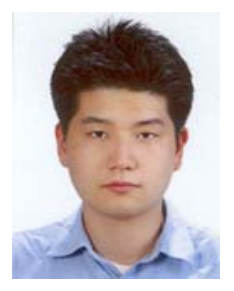

\section{고광은(Kwang-Eun Ko)}

2007년 : 중앙대학교 전자전기공학부 공학사 2007년 현재 : 중앙대학교 대학원 전자전 기공학부 석박사 통합과정

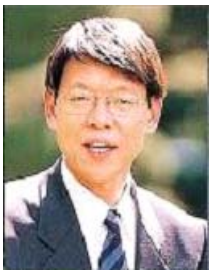

심귀보(Kwee-Bo Sim)

1990년: The University of Tokyo 전자공학과 공학박사

[제19권 5호 (2009년 10월호) 참조]
1991년 현재 : 중앙대학교 전자전기공학부 교수 2006년 2007년 : 한국지능시스템학회 회장

E-mail : kbsim@cau.ac.kr

Homepage URL: http://alife.cau.ac.kr

관심분야 : Multi-Agent Robotic Systems (MARS), Machine Learning Context Awareness, Emotion Recognition Systems

E-mail : kke@wm.cau.ac.kr 